\title{
Optimisation de la durée de traitement des pneumonies acquises sous ventilation mécanique
}

\author{
Optimization of duration of antimicrobial treatment of ventilatory-aquired pneumonia
}

\author{
J.-F. Timsit - S. Chemam - G. Voiriot $\cdot$ E. Mariotte $\cdot$ B. Mourvillier $\cdot$ J.-F. Soubirou $\cdot$ M. Neuville $\cdot$ R. Sonneville $\cdot$ \\ L. Bouadma $\cdot$ M. Wolff
}

Reçu le 13 janvier 2014 ; accepté le 29 janvier 2014

(C) SRLF et Springer-Verlag France 2014

Dans $80 \%$ des journées passées en réanimation, les patients reçoivent des antibiotiques. La principale raison motivant cette prescription en cours de séjour est la pneumonie nosocomiale acquise sous ventilation mécanique (PAVM) [1]. Or la surprescription d'antibiotiques peut entraîner des effets secondaires et peut induire la sélection de bactéries de moindre sensibilité aux antibiotiques dans le microbiote du patient traité, mais aussi dans l'écosystème que constitue le service de réanimation. Les antibiotiques doivent donc être prescrits à bon escient, pour une durée suffisante pour être efficaces, tout en minimisant les effets néfastes, en particulier écologiques. Pour limiter les durées de traitement, deux approches sont possibles et complémentaires : une approche globale fondée sur une durée minimale généralement efficace, et une approche individualisée basée sur le terrain, la gravité initiale de la symptomatologie, l'évolution dans les premiers jours des éléments cliniques et de certains paramètres biologiques comme la procalcitonine.

\section{Approche individualisée de limitation des durées de traitement des pneumonies nosocomiales}

Certaines études indiquent qu'une décision individualisée de l'arrêt des antibiotiques (utilisant par exemple des

J.-F. Timsit $(\bowtie) \cdot$ S. Chemam $\cdot$ G. Voiriot $\cdot$ E. Mariotte $\cdot$

B. Mourvillier · J.-F. Soubirou $\cdot$ M. Neuville $\cdot$ R. Sonneville

L. Bouadma $\cdot$ M. Wolff

CHU Bichat, Université Paris Diderot, réanimation médicale

et des maladies infectieuses, 46 rue Henri Huchard,

F-75018 Paris, France

e-mail : Jean-francois.timsit@bch.aphp.fr

IAME UMR 1137, Team 5,

DeSCID (Decision Sciences in Infectious Diseases) Prevention,

Control and Care, Université Paris Diderot, Inserm,

Sorbonne Paris Cité France éléments cliniques tels que la température ou les résultats d'un test sanguin) était associée à une plus courte durée de traitement et à une réduction globale de l'exposition aux antibiotiques, sans affecter négativement le devenir des patients.

Arrêt précoce de l'antibiothérapie basée sur l'évolution clinique

Des patients présentant une pneumonie, avec un score CPIS $\leq 6$ ont été randomisés pour recevoir trois jours de ciprofloxacine (400 $\mathrm{mg}$ x 3) (ou équivalent si le germe isolé de l'aspiration trachéale était résistant à la ciprofloxacine), arrêtée prématurément après trois jours si le $\mathrm{Cli}$ nical Pulmonary Infection Score (CPIS) restait inférieur à six, sinon avec un traitement long de 21 jours sans réévaluation [2]. Le traitement court n'a pas modifié la mortalité ni la durée de séjour, et a permis de limiter l'émergence de résistance ou une surinfection à germes résistants $(35 \% \mathrm{vs}$ $15 \% \mathrm{p}=0,017)$. Cette étude, arrêtée prématurément du fait de l'impact sur la résistance bactérienne, encourage un traitement très court des formes peu sévères de pneumonies acquises en réanimation ayant une évolution rapidement favorable.

En règle générale, le traitement prescrit est plus long lorsque le score CPIS est élevé. Cependant, une stratégie active de rediscussion d'un arrêt des antibiotiques par un intervenant extérieur se montre efficace [3]. Dans une étude monocentrique randomisée, où le diagnostic de PAVM était essentiellement clinique, Micek et al. ont comparé un rediscussion quotidienne de l'arrêt du traitement, basée sur l'évolution clinique et la recherche d'une étiologie alternative au sepsis, à la stratégie classique. L'intervention a permis une diminution de la durée d'antibiothérapie des PAVM $(6,0 \pm 4,9$ jours vs $8,0 \pm 5,6$ jours, $\mathrm{p}=0,001)$ sans impact sur le taux de récidive, la mortalité et la durée de séjour. 
Arrêt précoce du traitement des pneumonies cliniques basé sur les examens microbiologiques

L'arrêt précoce des antibiotiques au vu d'une culture de lavage alvéolaire $<10^{4} \mathrm{cfu} / \mathrm{ml}$ est associé à une diminution franche de la durée d'antibiothérapie (4 [3-4] vs 9 [6-14] jours) dans une analyse rétrospective d'un échantillon de 89 patients, et ceci sans impact sur la mortalité ni la durée de séjour. En comparaison des patients chez qui le traitement n'a pas été arrêté précocement malgré des résultats négatifs, ces patients présentaient moins d'infections respiratoires ainsi que non respiratoires, et moins d'infections à bactéries multirésistantes [4]. Ces résultats confirment des données anciennes obtenues avec la brosse bronchique protégée [5].

L'interprétation des résultats des études précédentes est délicate. On peut soit considérer que les patients inclus n'avaient pas de pneumonie et ne devaient donc pas recevoir d'antibiotiques, soit considérer que ces formes de pneumonie avec un inoculum faible, une gravité moindre et une évolution clinique rapidement satisfaisante relèvent d'un traitement très court. Cependant, quelle que soit l'interprétation, on peut conclure que des pneumopathies peu sévères ou pour lesquelles les prélèvements bactériologiques sont négatifs ou inférieurs aux seuils peuvent relever d'un traitement très court. Ce raisonnement n'est valable et possible que si des prélèvements bactériologiques sont effectués avant toute instauration d'un traitement antibiotique empirique [6].

\section{Arrêt précoce basé sur l'évolution de biomarqueurs}

L'arrêt précoce basé sur l'évolution clinique est une décision parfois délicate car les signes cliniques peuvent être discordants. Dans un telle démarche, l'apport de la baisse de la procalcitonine comme marqueur objectif d'évolution favorable des infections sévères est suggéré par plusieurs essais randomisés [7,8] et des méta-analyses [9-11]. Dans notre expérience, l'utilisation de la chute de plus de $85 \%$ de la procalcitonine pour décider de l'arrêt du traitement des PAVM aboutissait à une réduction de l'utilisation des antibiotiques de plus de 2 jours (7,3 vs 9.4 jours) sans impact négatif sur la mortalité, la durée de séjour et la durée de ventilation mécanique [8].

\section{Arrêt précoce basé sur l'évolution de la colonisation bronchique}

La stérilisation des prélèvements bronchiques évoque une évolution favorable. Cependant, à l'inverse, la persistance de prélèvements bronchiques positifs, en particulier à $P$. aeruginosa et à d'autres bacilles à Gram négatif (BGN) non fermentant, ne signe pas l'échec thérapeutique mais la colonisation résiduelle. Celle-ci ne doit être traitée qu'en cas d'évolution clinique défavorable [12].

\section{Approche globale de limitation des durées de traitement}

Une autre approche consiste à réduire de façon globale la durée de traitement des pneumonies, en particulier celles acquises sous ventilation mécanique. L'étude Pneuma randomisée contrôlée a montré que pour des PAVM survenant chez des patients de gravité intermédiaire non immunodéprimés, un traitement raccourci à huit jours était aussi efficace, en termes de mortalité, de durée de ventilation mécanique et de durée de séjour qu'un traitement de 15 jours [13]. Des résultats similaires ont été obtenus dans le traitement des PAVM précoces [14]. Cependant, le traitement court des PAVM précoces est associé à une plus grande fréquence d'infections secondaires et à un taux de rechutes plus élevé dans les PAVM tardives à BGN non fermentant, sans toutefois impacter le pronostic et la durée de séjour $[13,14]$.

\section{Chez quels patients la limitation des durées de traitement doit-elle être envisagée avec prudence?}

Il convient de garder en mémoire que les patients neutropéniques [13,14], atteints de mucoviscidose [14], immunodéprimés [13], n'étaient pas inclus dans ces essais randomisés. La population des pneumonies nosocomiales tardives incluse dans l'étude Pneuma [13] ne représentait que 482 des 991 épisodes survenus. En particulier, les patients très sévères (indice de gravité simplifié 2, IGS2 à l'inclusion > 65) n'étaient pas éligibles. Enfin, les patients devaient recevoir immédiatement une antibiothérapie adéquate, le plus souvent une bi-antibiothérapie.

Donc chez les patients à haut risque de récidive, c'està-dire les patients âgés, plus sévères, immunodéprimés, ayant une PAVM à $\mathrm{S}$. aureus méticilline-résistant ou à BGN non fermentant, ayant une évolution initiale moins rapidement favorable $[15,16]$, le traitement court doit être réfléchi au cas par cas $[15,16]$. Enfin, s'il existe un empyème ou un abcès, le traitement court n'est pas souhaitable [14].

\section{Conclusion}

La limitation de la durée de l'antibiothérapie est un élément clé de la lutte contre la résistance des bactéries aux antibiotiques. La durée de traitement des PAVM peut être limitée à huit jours lors du premier épisode, si le traitement est immédiatement efficace et l'évolution rapidement favorable. Elle peut même être plus courte en cas de formes cliniques peu sévères non confirmées bactériologiquement,

En cas de PAVM tardive à BGN non fermentant, survenant chez des patients à risque de récidive, un arrêt précoce 
du traitement doit être envisagé avec prudence, en se basant notamment sur l'évolution clinique favorable et la baisse substantielle de la procalcitonine.

Conflit d'intérêt : J.-F. Timsit, S. Chemam, G. Voiriot, E. Mariotte, B. Mourvillier, J.-F. Soubirou, M. Neuville, R. Sonneville et L. Bouadma déclarent ne pas avoir de conflit d'intérêt.

Michel Wolff : conférences ou participation à un board pour Astellas, Gilead, AstraZeneca et Menarini.

\section{Références}

1. Zahar JR, Nguile-Makao M, Francais A, et al (2009) Predicting the risk of documented ventilator-associated pneumonia for benchmarking: construction and validation of a score. Crit Care Med 37:2545-51

2. Singh N, Rogers P, Atwood CW, et al (2000) Short-course empiric antibiotic therapy for patients with pulmonary infiltrates in the intensive care unit. A proposed solution for indiscriminate antibiotic prescription. Am J Respir Crit Care Med 162:505-11

3. Micek ST, Ward S, Fraser VJ, Kollef MH (2004) A randomized controlled trial of an antibiotic discontinuation policy for clinically suspected ventilator-associated pneumonia. Chest 125: 1791-9

4. Raman K, Nailor MD, Nicolau DP, et al (2013) Early antibiotic discontinuation in patients with clinically suspected ventilatorassociated pneumonia and negative quantitative bronchoscopy cultures. Crit Care Med 41:1656-63

5. Bonten MJ, Bergmans DC, Stobberingh EE, et al (1997) Implementation of bronchoscopic techniques in the diagnosis of ventilator-associated pneumonia to reduce antibiotic use. Am J Respir Crit Care Med 156:1820-4
6. Timsit JF (2007) Bronchoalveolar lavage for VAP diagnosis: patients must be sampled before any change of antimicrobial therapy. Intensive Care Med 33:1690-3

7. Nobre V, Harbarth S, Graf JD, et al (2008) Use of procalcitonin to shorten antibiotic treatment duration in septic patients: a randomized trial. Am J Respir Crit Care Med 177:498-505

8. Bouadma L, Luyt CE, Tubach F, et al (2010) Use of procalcitonin to reduce patients' exposure to antibiotics in intensive care units (PRORATA trial): a multicentre randomised controlled trial. Lancet 375:463-74

9. Matthaiou DK, Ntani G, Kontogiorgi M, et al (2012) An ESICM systematic review and meta-analysis of procalcitonin-guided antibiotic therapy algorithms in adult critically ill patients. Intensive Care Med 38:940-9

10. Schuetz P, Briel M, Christ-Crain M, et al (2012) Procalcitonin to guide initiation and duration of antibiotic treatment in acute respiratory infections: an individual patient data meta-analysis. Clin Infect Dis 55:651-62

11. Kopterides P, Siempos, II, Tsangaris I, et al (2010) Procalcitoninguided algorithms of antibiotic therapy in the intensive care unit: a systematic review and meta-analysis of randomized controlled trials. Crit Care Med 38:2229-41

12. Dennesen PJ, van der Ven AJ, Kessels AG, et al (2001) Resolution of infectious parameters after antimicrobial therapy in patients with ventilator-associated pneumonia. Am J Respir Crit Care Med 163:1371-5

13. Chastre J, Wolff M, Fagon JY, et al (2003) Comparison of 8 vs 15 days of antibiotic therapy for ventilator-associated pneumonia in adults: a randomized trial. JAMA 290:2588-98

14. Capellier G, Mockly H, Charpentier C, et al (2012) Early-onset ventilator-associated pneumonia in adults randomized clinical trial: comparison of 8 versus 15 days of antibiotic treatment. PLoS One 7:e41290

15. Combes A, Luyt CE, Fagon JY, et al (2007) Early predictors for infection recurrence and death in patients with ventilatorassociated pneumonia. Crit Care Med 35:146-54

16. Planquette B, Timsit JF, Misset BY, et al (2013) Pseudomonas aeruginosa ventilator-associated pneumonia. predictive factors of treatment failure. Am J Respir Crit Care Med 188:69-76 